\title{
Paranephric abscess secondary to perforated appendicitis; an abdominal drainage is a rule rather than an option during appendectomy
}

\author{
Asri Che Jusoh*, Nasturah Abdullah, and Ong Yanzie \\ Department of General Surgery, Kuala Krai Hospital, 18000 Kelantan, Malaysia
}

\begin{abstract}
Acute appendicitis is the most common acute surgical condition of the abdomen. Unfortunately, atypical presentations are common. Delayed diagnosis would therefore lead to higher morbidity and mortality. Furthermore, under estimation of an associated retro-peritoneal abscess can be disastrous. Our case illustrates the issues mentioned. A young lady presented with atypical symptoms of appendicitis but was diagnosed as perforated retrocaecal appendicitis with paranephric abscess following CT abdomen. An open appendectomy and drainage of retroperitoneal abscess were done with no drain inserted. Unfortunately four days later she developed a complete wound failure with surgical site infection. A diagnostic laparoscopy and drainage of retroperitoneal abscess was subsequently done. Post operatively, a total of $2000 \mathrm{mls}$ of pus evacuated in 10 days. She recovered well and was discharged on post-operative day 14 . Our case illustrates the atypical presentation of complicated retro-caecal appendicitis with retro-peritoneal (para-renal) abscess. A CT scan prior surgery is extremely beneficial in order to determine the best surgical approach and amount of retro-peritoneal abscess noted during an index surgery would be misleading. Therefore, a drain must always be inserted in order to avoid future morbidities.
\end{abstract}

\section{Introduction}

Acute appendicitis is one of the important differential diagnoses in patient with acute abdomen. Unfortunately, it has many unusual presentations [1]. The classic history of anorexia and peri-umbilical pain followed by nausea, right lower quadrant (RLQ) pain, and vomiting occurs in only $50 \%$ of cases [2]. Furthermore, position of the appendix varies. Its base located at the Mc Burney's point in only $4 \%$ of patients and nearly $40 \%$ were more than $5 \mathrm{~cm}$ from the point based on 3-D MDCT studies [3]. In addition to that, retro-caecally positioned appendix was common $(65 \%)$ which frequently extraperitoneally located $[4,5]$. Complicated retro-caecal appendicitis may leads to retroperitoneal abscess [6]. In such cases early diagnosis is difficult as insidious presenting symptoms with subtle physical findings lead to higher morbidity and mortality $[7,8]$. As a result, infections can progress cranially to involve both anterior and posterior para-nephric spaces.

Our case demonstrates atypical presentation of complicated retrocaecal appendicitis and morbidities developed. We believe the latter developed due to under-estimation of volume of pus during initial surgery. A drain inserted during index surgery could have save her from most post-operative morbidities.

\section{Case}

A 20-year-old lady presented with three days history of right sided abdominal pain and vomiting. She also experienced anorexia with minimal bowel opening. Clinically, she was febrile $\left(38^{\circ} \mathrm{C}\right)$ and appeared septic. Her abdomen was distended with tenderness only on deep palpation at right lower quadrant without muscle rigidity or mass. Laboratory investigations including WBC count was surprisingly normal $\left(9.05 \times 10^{3} / \mathrm{uL}\right)$. Her calculated RIPASA (Raja Isteri Pengiran
Anak Saleha) and ALVARADO score was only 7.5 and 5 respectively. She was empirically started with intravenous Cefoperazone and Metronidazole.

Her abdominal X-ray shows generalized dilatation of small bowel with air fluid level. Complicated retro-caecal appendicitis with ileus was suspected by which a CT scan was requested prior to surgery. The latter demonstrates a dilated retro-caecal appendix with appendicolith indicative of perforated acute appendicitis. Interestingly, there were also multiple air pockets and fluid collection suggestive of pus at right anterior and posterior para-renal spaces and at right iliac fossae, surrounding the appendix (Figures 1 and 2). It was estimated around $250 \mathrm{ml}$. The small bowel and cecum were dilated with air fluid level but there was no obvious intra-peritoneal collection (Figure 3).

A diagnosis of acute perforated appendicitis with retroperitoneal abscess was made and she underwent an open appendectomy and drainage via right lower transverse incision. The latter was thought feasible rather than a midline approach as no intra-peritoneal soiling and localized abscess on CT scan. A fragile and perforated retro-caecal appendix was noted during surgery. Its body and tip were within retroperitoneal space with a fecalith within. Most of the pus was retroperitoneally located. The wound has to be extended laterally to facilitate mobilisation of caecum and ascending colon medially. Subsequently,

Correspondence to: Asri Che Jusoh, Consultant General Surgeon, Department of General Surgery, Kuala Krai Hospital, 18000 Kelantan, Malaysia, Tel: +6099666202; E-mail: asricj@yahoo.com

Key words: appendicitis, retroperitoneal abscess, paranephric abscess

Received: December 14, 2015; Accepted: January 22, 2016; Published: January 26,2016 


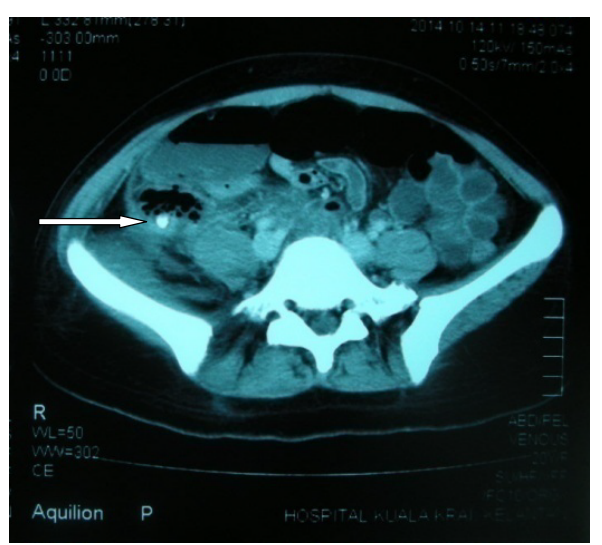

Figure 1. Axial $\mathrm{CT}$ at the level of caecum demonstrates an appendicolith at right iliac fossae (white arrow).

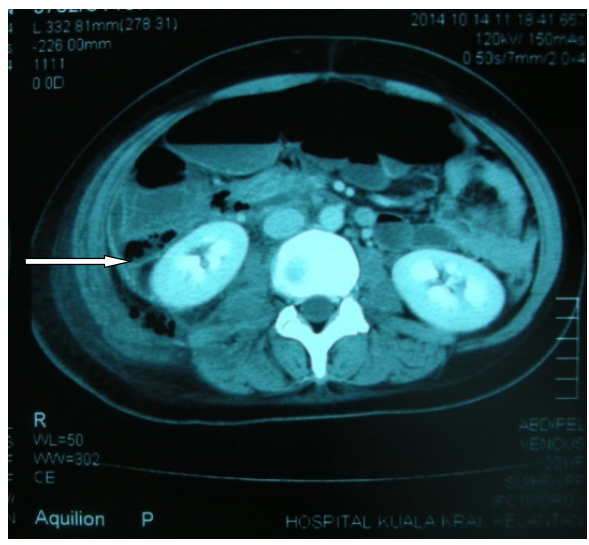

Figure 2. Multiple air pockets and fluid collection suggestive of pus at right anterior and posterior para-renal spaces and at right iliac fossae

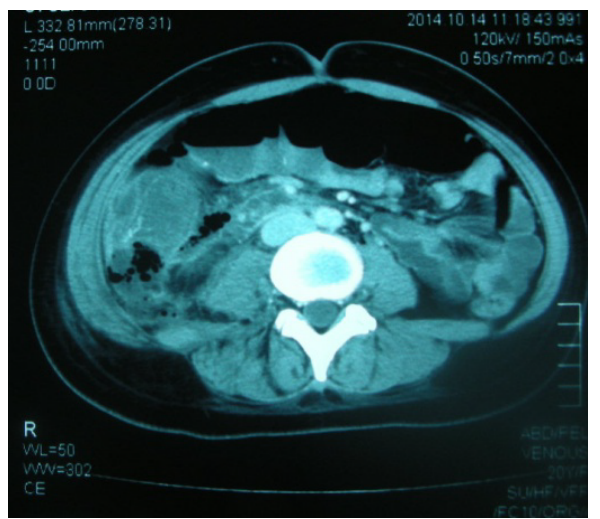

Figure 3. Small bowel and cecum are dilated with air fluid level but there was no obvious intra-peritoneal collection.

her appendix was removed and about $200 \mathrm{ml}$ of retroperitoneal pus evacuated. The latter space appeared dry prior closure of the abdomen hence no drain inserted. The antibiotics were continued as pus culture grew sensitive E.coli.

Unfortunately, four days later she developed a new onset of sepsis with pus draining out from the wound. A diagnosis of complete wound failure with SSI (surgical site infection) was made as part of small bowel was noted following suture removal. Otherwise she had no peritonitis. An urgent ultrasound abdomen showed fluid collections in the pelvic cavity and both right and left iliac fossa with internal echoes.

A diagnostic laparoscopy and repair of abdominal wall dehiscence were subsequently performed. Intra-operatively, no obvious abscesses seen elsewhere other than at right para-colic area. Fifty $\mathrm{ml}$ of pus was evacuated and a drain inserted retroperitoneally. Unexpectedly, average of $200-300 \mathrm{ml}$ of pus drained every day before it dries up a week later. Her antibiotic was upgraded to Meropenem while Metronidazole continues as pus culture growth E.coli and was sensitive to Carbapenem group.

Her post-operative period was quite uneventful. Unexpectedly, a total of $2200 \mathrm{ml}$ of pus was drained in 10 days' time (average of $200-$ $250 \mathrm{ml}$ per day). She was subsequently discharged on post-operative day 14.

\section{Discussion}

A diagnosis of retroperitoneal abscess (RPA) is often delayed or missed as onset of symptoms typically slow with limited localizing signs $[7,9,10]$. The duration of symptoms prior diagnosis typically weeks to months and most common symptom such as pain, frequently at lower abdomen and flank [7]. Therefore, careful history taking and high index of suspicion required for diagnosis ${ }^{[8]}$. Fortunately in our case, her presentation was not that atypical of appendicitis except minimal abdominal signs. However, associated RPA was really unexpected.

Fifty percent of RPA occur at anterior retroperitoneal space and most were due to diverticulitis, appendicitis or perforated colon cancer [7]. Furthermore, Raymond et al. [11] demonstrated that, $18 \%$ of 84 cases of anterior para-nephric abscess were appendix related [11]. Perforation of extra-peritoneal appendix or abscess can extend retroperitoneally to both anterior and posterior para-nephric spaces as both communicate inferiorly [5,7]. Until 2010, only 26 cases of such have been reported in English literature [9].

Radiological examinations play a major role in the diagnosis of extra-peritoneal infection or abscess due to unreliable clinical findings and laboratory results. Plain abdominal X-ray occasionally revealed obliteration of psoas shadow or gas bubbles overlying right abdominal wall [12]. Nevertheless, CT scan is preferred as it offers additional advantages of determining the extent and possible site of origin of abscess [12]. Indeed, its findings will determine the best surgical approach. Despite that, we should acknowledge of a low sensitivity of CT scan (42\%) in identifying a perforated appendicitis as a cause of RPA. Severe inflammation made the appendix becomes necrotic and indistinguishable from abscess on CT scan [13]. Fortunately, in ours it can be seen quite well.

Most authors embarked on open surgery rather than percutaneous drainage of RPA as complicated appendicitis can be removed concurrently. Interestingly, midline laparotomy was the preferred approach $[1,6,13]$. Some authors even approached the abscess retroperitoneally first before proceed with appendectomy for complicated appendicitis as the latter was not suspected initially [11]. In contrast to others, we approached it via transverse right lower quadrant incision as there was no intra-peritoneal soiling and the pus was localized underneath the ascending colon. The latter can be mobilized quite easily to facilitate evacuation of RPA. Unfortunately we underestimate residual abscess retro-peritoneally hence no drain was inserted.

It is well established by meta-analysis of randomized controlled trials (RCT) that prophylactic drain should be avoided in any stage of appendicitis. However, none were associated with RPA [14-16]. 
The latter has few peculiar features which we believe mandate routine drainage. Peri-nephric abscess of renal origin typically confines to Gerota's fascia which often small in volume. In stark contrast, paranephric abscess usually large in volume (up to $2000 \mathrm{mls}$ ) and most surgeons could not define its compartment containing pus [10]. Absence of drain leads to rapid re-accumulation of pus and worsening sepsis such in ours.

\section{Conclusion}

Our case has thought us few important lessons other than its atypical presentation. A CT scan prior surgery would definitely determine the best approach with least morbidity. Not all patients benefited from midline laparotomy. Drainage of RPA is considered mandatory which can be done trans-abdominally during an appendectomy or retroperitoneally.

\section{Authors' contribution} paper.

Asri che Jusoh involved in designing, compiling and writing the

Nasturah Abdullah participated in doing literature search and organizing the reviewed paper.

Ong Yanzie involved in acquisition of data and prepares the case history.

All authors have read the paper and approved the final manuscript.

\section{Competing interests}

Asri Che Jusoh, Nasturah Abdullah and Ong Yanzie declare that we have no competing interests either financially or non-financially.

\section{References}

1. Fanning DM, Barry M, O'Brien GC, Leahy AL (2007) Perforation of a retrocaecal appendix presenting clinically as a right lumbar abscess. Surgeon 5: 368-370. [Crossref]

2. Yeh B (2008) Evidence-based emergency medicine/rational clinical examination abstract. Does this adult patient have appendicitis? Ann Emerg Med 52: 301-303. [Crossref]
3. Oto A, Ernst RD, Mileski WJ, Nishino TK, Le O, et al. (2006) Localization of appendix with MDCT and influence of findings on choice of appendectomy incision. AJR Am J Roentgenol 187: 987-990. [Crossref]

4. Kim S, Lim HK, Lee JY, Lee J, Kim MJ, et al. (2006) Ascending retrocecal appendicitis: clinical and computed tomographic findings. J Comput Assist Tomogr 30: 772-776. [Crossref]

5. Kousei I, GeetikaK, Isaac SLD, Yutaka S (2004) Gas-forming abdominal wall abscess: unusual manifestation of perforated retroperitoneal appendicitis extending through the superior lumbar triangle. Emergency Radiology. 10: 207-209.

6. Behrang L, Alicia S. H, David JL, Maria S (2013) Perforation of a Retrocecal Appendix Resulting in Retroperitoneal Air: A Case Report. Case Reports in Radiology.

7. Edwards JD, Eckhauser FE (1986) Retroperitoneal perforation of the appendix presenting as subcutaneous emphysema of the thigh. Dis Colon Rectum 29: 456-458. [Crossref]

8. Kao CT, Tsai JD, Lee HC, Wang NL, Shih SL, et al. (2002) Right perinephric abscess: a rare presentation of ruptured retrocecal appendicitis. PediatrNephrol 17: 177-180. [Crossref]

9. Michele Diana, AlexandreParoz, Nicolas Demartines, Markus Schäfer (2010) Retroperitoneal abscess with concomitant hepatic portal venous gas and rectal perforation: a rare triad of complications of acute appendicitis. A case report World $J$ EmergSurg 5: 3. [Crossref]

10. Crepps JT, Welch JP, Orlando R (1987) Management and outcome of retroperitoneal abscesses. Ann Surg 205: 276-281. [Crossref]

11. Raymond AB, Kevin PK, Gregory GD, Jack LS, Joel AP (1996) Acute appendicitis presenting as a perinephric abscess. The journal of urology 156: 1758 .

12. McGahan JP (1982) Perinephric abscess secondary to ruptured retrocecal appendix diagnosed by computerized tomography. Urology 19: 217-219. [Crossref]

13. Hsieh CH, Wang YC, Yang HR, Chung PK, Jeng LB, et al. (2007) Retroperitoneal abscess resulting from perforated acute appendicitis: analysis of its management and outcome. Surg Today 37: 762-767. [Crossref]

14. Petrowsky H, Demartines N, Rousson V, Clavien PA (2004) Evidence-based value of prophylactic drainage in gastrointestinal surgery: a systematic review and metaanalyses. Ann Surg 240: 1074-1084. [Crossref]

15. Cheng Y, Zhou S, Zhou R, Lu J, Wu S, et al. (2015) Abdominal drainage to prevent intra-peritoneal abscess after open appendectomy for complicated appendicitis. Cochrane Database Syst Rev 2: CD010168. [Crossref]

16. Rather SA, Bari SU, Malik AA, Khan A (2013) Drainage vs no drainage in secondary peritonitis with sepsis following complicated appendicitis in adults in the modern era of antibiotics. World J GastrointestSurg 5: 300-305. [Crossref]

Copyright: (C2015 Jusoh AC. This is an open-access article distributed under the terms of the Creative Commons Attribution License, which permits unrestricted use, distribution, and reproduction in any medium, provided the original author and source are credited. 